\title{
DESIGN E TECNOLOGIA: UMA ABORDAGEM BIBLIOMÉTRICA NO PERIÓDICO DESIGN STUDIES.
}

\section{DESIGN AND TECHNOLOGY: A BIBLIOMETRIC RE- VIEW IN THE DESIGN STUDIES JOURNAL.}

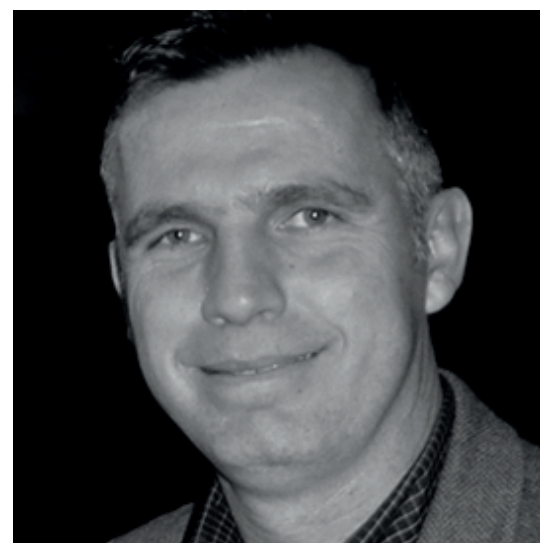

\section{Luis Carlos Paschoarelli}

Pós-doutorado em Ergonomia pela Universidade

Técnica de Lisboa

Livre-Docente em Design Ergonômico pela Universidade

Estadual Paulista

paschoarelli@faac.unesp.br

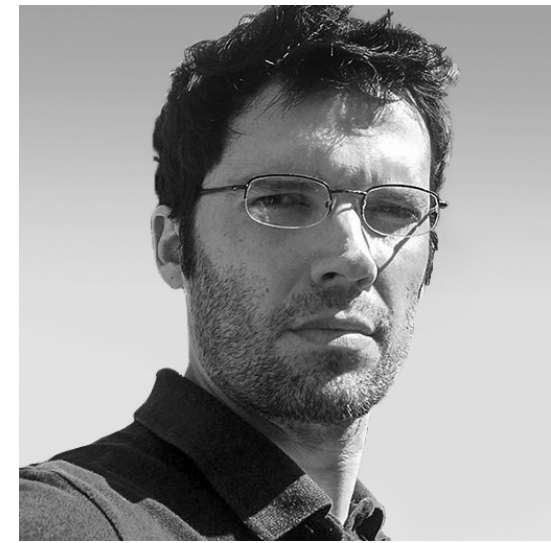

\section{Fabrício Mira}

Mestre em Design pela Universidade Estadual Paulista Júlio de Mesquita Filho (UNESP)

Programa de Pós-Graduação em Design (UNESP)

fabriciomira@gmail.com 


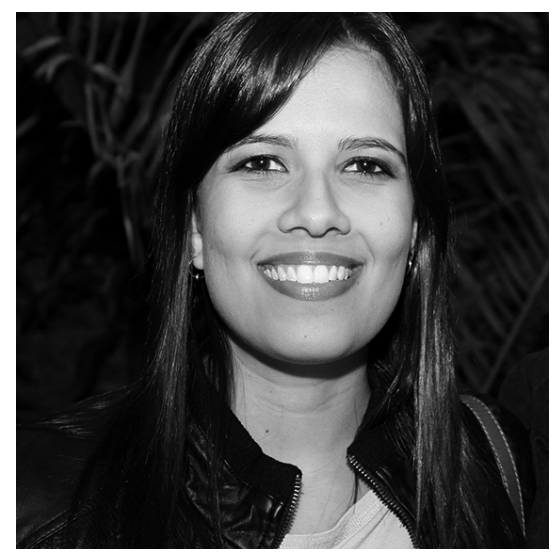

\section{Lílian Lago}

Mestre em Design pela Universidade Estadual Paulista Júlio de Mesquita Filho (UNESP)

Programa de Pós-Graduação em Design (UNESP) lago.Iilian@gmail.com

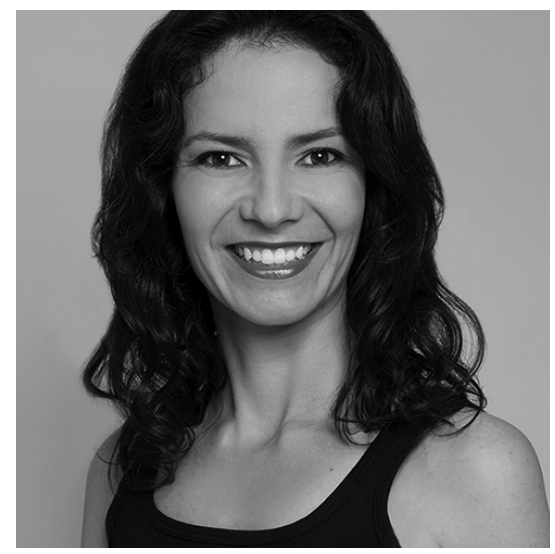

Juliana Cristina de Angelo

Mestre em Design pela Universidade Estadual Paulista Júlio de Mesquita Filho (UNESP) Programa de Pós-Graduação em Design (UNESP) jul.angel@hotmail.com

\section{RESUMO}

As novas tecnologias de produção e informação estão cada vez mais presentes no cotidiano de bilhões de usuários no mundo todo, gerando uma demanda pela atuação de designers na relação usuário/tecnologia. Neste contexto, o objetivo deste estudo foi analisar a importância da tecnologia nos estudos em design, considerando como objeto de análise os artigos publicados no periódico internacional Design Studies. Os procedimentos metodológicos foram realizados a partir da base de dados Scopus e por meio do software de análises bibliométricas VOSviewer, resultando na identificação dos artigos que relacionaram a tecnologia e o design em sua temática. Os resultados indicam um crescimento progressivo da produção científica sobre o tema, com ênfase na abordagem da tecnologia como ferramenta no projeto, particularmente na geração de ideias, na resolução de problemas e no trabalho em equipe. Ao mesmo tempo, observou-se uma carência de artigos sobre o uso da tecnologia pelo público em geral, revelando uma lacuna do estudo sobre a interação usuário/tecnologia 
diante de um cenário de expansão do uso de tecnologias virtuais nos sistemas de produção e informação.

\section{PALAVRAS-CHAVE}

Design; Interface; Tecnologia.

\section{ABSTRACT}

Production and information technologies are increasingly present in the daily lives of billions of users worldwide, generating a demand by the intervention of designers on user-technology relationship. In this context, the purpose of this study was to analyze the evolution of technology in research in design, considering as object of analysis articles published in the international journal Design Studies. The methodological procedures were performed in the Scopus database and bibliometric analysis software VOSviewer, resulting in the identification of articles that related technology and design in their subject. The results indicate a progressive growth of scientific literature on the topic, emphasizing the approach of technology as a tool for designers in project phase, particularly in the generation of ideas, problem solving and teamwork. However, it was noticed a lack of articles about the use of technology by general public, revealing a need of research on user-technology interaction considering an expansion scenario for virtual technologies in production and information systems.

\section{KEYWORDS}

Design; Interface; Technology.

\section{INTRODUÇÃO}

O desenvolvimento tecnológico é um dos objetos de análise mais discutidos de todos os tempos, especialmente ao se considerar o quanto seu aperfeiçoamento tem influenciado o modo de vida e as características culturais no mundo todo. Tal influência decorre, especialmente, de fatos ocorridos após a 2a Guerra Mundial, quando a tecnologia começou a se tornar factível nos mais diferentes aparatos de uso cotidiano.

As novas tecnologias de produção e informação ganharam espaço, promovendo uma reorganização da produção e distribuição de artefatos, informações e conteúdos, bem como mudanças nas práticas e rotinas profissionais e sociais, influenciando decisivamente o design de produtos, sistemas e informação. Para McGarry (1999), os avanços tecnológicos se desenvolvem a partir da necessida- 
de de comunicação entre os homens e da interação com o ambiente. E quando se trata da estrutura ambiental urbana, desenvolvida especialmente a partir de meados do século XX, a interação entre os usuários e os produtos, aplicações e sistemas tecnológicos se dá por meio do design.

Com o propósito de investigar a importância da tecnologia para a construção do conhecimento na área do design, este estudo apresenta uma revisão bibliométrica dos artigos publicados sobre tecnologia no periódico Design Studies entre 1979 e 2015. Identifica as principais universidades e autores deste período e analisa o crescimento dos estudos sobre o tema diante de um cenário de expansão do uso de tecnologias virtuais, tanto nos sistemas de produção, quanto nos sistemas de informação.

\section{SOCIEDADE, DESIGN E TECNOLOGIA}

De acordo com De Masi (2000), a sociedade passa por uma mudança de época quando três inovações distintas coincidem: novas fontes energéticas, novas divisões do trabalho e novas divisões do poder. A primeira época considerada na história da evolução do homem social é a "era rural", que se deu a partir do desenvolvimento da agricultura e da pecuária. O homem deixou de ser nômade para se fixar à terra e gerar riquezas. O processo produtivo era fruto do trabalho manual e rudimentar do homem e dos animais.

A segunda era, chamada "industrial" ou "moderna", teve seu início no século XVIII nos países desenvolvidos e foi caracterizada e concebida pela industrialização. A sociedade se uniu, estabeleceu grandes organizações coletivas - as fábricas, os partidos e os sindicatos, as cidades - e consolidou-se como um universo linear, programado e padronizado, repleto de produtos provenientes do trabalho mecânico de uma massa de trabalhadores liderada sob o frenesi da precisão.

Mecanização, sincronização, padronização, maximização, especialização, centralização e concentração regiam tanto o sistema produtivo quanto a vida social e familiar. A ciência moderna, apesar de sua fragmentação, avançou de forma exponencial. O progresso baseado nas especializações foi indiscutível por produzir avanços em todos os campos do conhecimento científico e da técnica. Entretanto, a sociedade industrial não perdurou por muito tempo além

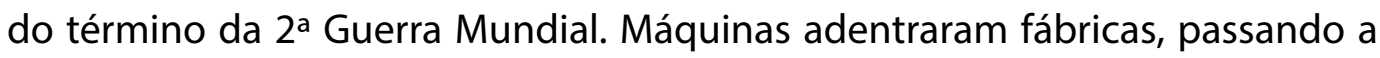
executar boa parte do trabalho antes feito de forma manual; a economia de serviços se sobressaiu em relação à produção de bens; as taxas de escolarização aumentaram, entre outros acontecimentos que culminaram numa nova era da 
informação e da comunicação. Esta nova era pode ser chamada de "sociedade pós-industrial" - a sociedade contemporânea.

A evolução da ciência, das técnicas e da tecnologia pode ser considerada intrínseca às inovações que culminam nas mudanças de época. O desenvolvimento da tecnologia costumou estar associado a um aparato físico. Todavia, as tecnologias virtuais são as que mais impactam a sociedade (RIBEIRO; SILVEIRA, 2013). Todos os campos pertencentes à sociedade atual estão sob a influência das novas tecnologias de informação e de comunicação, caracterizando esta sociedade como "informacional" (LIPOVETSKY; SERROY, 2011).

O International Council of Societies of Industrial Design define o design como uma atividade criativa cujo objetivo é estabelecer as qualidades multifacetadas dos objetos, processos, serviços e seus sistemas em todo o ciclo de vida. Portanto, o design é o fator central da humanização inovadora de tecnologias e o fator crucial de intercâmbio cultural e econômico (ICSID, 2015).

Bonsiepe (2012, p. 19) aponta que o design se posiciona na intersecção entre a cultura da vida cotidiana, da tecnologia e da economia - um lugar que pode ser chamado de "o mundo da vida cotidiana". O caráter operacional dos artefatos materiais e semióticos é o foco do design, que não interpreta a função e a funcionalidade em termos de eficiência física, como faz a engenharia, mas sim em termos de comportamento incorporado a uma dinâmica cultural e social. $\mathrm{O}$ design deve integrar ciência e tecnologia na vida cotidiana, enfocando a intersecção entre o usuário e o produto/ informação — o chamado "design de interface".

O cenário tecnológico atual apresenta dois importantes fenômenos de existência diretamente relacionada ao design - a "internet das coisas" (Internet of Things - loT) e "internet de todas as coisas" (Internet of Everything - loE). Evans, autor do white paper de abril de 2011, da Cisco IBSG (EVANS, 2011, p. 6), descreve a loT como a rede das redes - "um salto que levará a aplicações revolucionárias com potencial para melhorar consideravelmente a forma como as pessoas vivem, aprendem, trabalham e se divertem". O autor estima que a loT nasceu entre 2008 e 2009, quando o número de dispositivos conectados à internet superou a população mundial. Em 2003, a população mundial era de 6,3 bilhões e os dispositivos conectados eram 500 milhões; enquanto em 2010, a população era de 6,8 bilhões e os dispositivos conectados 12,5 bilhões. Os números são resultado, em maior parte, do crescimento exponencial das vendas de smartphones e tablets.

Para o European Research Cluster on the Internet of Things (IERC, 2015), a IoT tem como objetivo integrar o mundo físico com o virtual, usando a inter- 
net como meio de comunicação e troca de informações. O desenvolvimento das tecnologias de informação e comunicação geram cada vez mais objetos com capacidade para se comunicar com outros objetos, capacitando-os como participantes ativos que transformam o mundo físico em uma fonte de informação.

De acordo com Singer (2012), o nascimento da expressão loT envolve pesquisadores do Auto-ID Center e do Massachusetts Institute of Technology. Ashton (2009) afirma ter cunhado a expressão em uma apresentação de trabalho na Procter \& Gamble em 1999 para falar de tarefas realizadas por computadores e RFID - radio frequency identification. Brock (2001) publica o termo em uma edição do white paper da Auto-ID Center para descrever um cenário onde uma infraestrutura inteligente iria conectar objetos por meio de uma rede de computadores de forma remota. E, para tanto, o autor afirma ter como objetivo criar padrões, protocolos e linguagens para facilitar a adoção mundial desta rede, que viria a formar a internet das coisas.

A International Telecommunication Union (ITU, 2005), agência da ONU especializada em tecnologias de informação e comunicação, publica relatórios anuais sobre as tecnologias emergentes e, no ano de 2005, a loT tornou-se pauta da publicação.

A partir de 2013, a Cisco IBSG passou a utilizar o termo "internet de todas as coisas" (Internet of Everything - loE). Para Evans (2011), a loT se baseia na conexão de objetos, enquanto a loE integra pessoas (usuários, seres humanos), processos (gerencia a maneira como pessoas, dados e coisas cooperam), dados (informação) e coisas (objetos e dispositivos inanimados) para fazer conexões em rede mais relevantes do que aquelas feitas anteriormente, transformando informações em ações que, por sua vez, criam experiências mais ricas e oportunidades econômicas para as empresas, indivíduos e países. O autor ainda aponta que os relatórios da Cisco preveem que a loE tem potencial para fazer 50 bilhões de conexões entre pessoas, dados e coisas até o ano de 2020.

Bradley, Barbier e Handler (2013) argumentam que a loE é impulsionada por diversos fatores, como o aumento das capacidades de processamento, armazenamento e largura de banda, o rápido crescimento da computação em nuvem, mídia social e móvel e a maior capacidade para combinar tecnologias (hardware e software) de forma eficiente a fim de tirar maior proveito da conectividade. Os autores também descrevem a dimensão do mercado a ser explorado pela loE por meio de uma estatística: 99,4\% dos objetos físicos que tem capacidade para serem conectados à internet ainda estão desconectados. 
Para Chambers (2012), a loE tem a inteligência de rede como um importante facilitador, responsável por alimentar as capacidades de gerenciamento, controle e escalabilidade necessárias para suportar o crescimento exponencial do número de conexões. $\mathrm{O}$ autor não deixa de mencionar a presença de riscos relacionados à segurança e à privacidade num mundo hiper-conectado, mas incentiva que estes sejam enfrentados e que se trabalhe para que todo desenvolvimento resulte em um saldo positivo para a sociedade.

A loE avança para promover melhores resultados para o varejo, os serviços e o setor produtivo. São sistemas fabris inteligentes, marketing e propaganda conectados, jogos e entretenimento, prédios com aquecimento, ventilação, segurança e iluminação inteligentes, veículos conectados, serviços de saúde e monitoramento de pacientes, sistemas para monitoramento de animais e inúmeros outros projetos (BRADLEY; BARBIER; HANDLER, 2013; BRADLEY et al., 2015).

Desta forma, pode-se compreender que a loE permite a comunicação e colaboração entre máquinas e pessoas, configurando uma nova era da computação e da comunicação que veio para transformar de forma radical as esferas pessoal, comunitária e corporativa. A partir das constatações do ICSID (2015) e Bonsiepe (2012) vê-se que o design é uma atividade necessária para a humanização da tecnologia por meio do projeto de interfaces adequadas e facilitadas, pois a interface é o suporte que permite a interação entre usuários, objetos, dados e processos. O desenvolvimento exponencial da loE se apresenta como atrelado à necessidade de pesquisa e desenvolvimento na área do design, em especial o design de interface.

\section{INTERAÇÃO E INTERFACE}

As primeiras interfaces computacionais, criadas na década de 1940 consistiam em alavancas, chaves e lâmpadas por meio das quais era feita a comunicação com o computador, baseada em linguagem de máquina. $O$ homem tinha que adaptar seu comportamento às máquinas por meio de um treinamento simbólico e abstrato (BURDEA; COIFFET, 1994; BONSIEPE, 1999). Os anos que sucedem a $2^{\text {a }}$ Guerra Mundial são marcados pelo funcionalismo, movimento racionalista disseminado pela Escola Superior de Design de Ulm, na Alemanha, cujo princípio "a forma segue a função" direcionava o foco do design ao objeto. Nas décadas seguintes, pode-se dizer que as máquinas evoluíram no sentido de se ajustarem ao homem por meio de interfaces cada vez mais intuitivas. Isto se deu graças ao desenvolvimento da tecnologia, que permitiu o surgimento de soluções cada vez mais amigáveis, aliado à mudança da mentalidade projetual: 
o design pós-moderno se volta para o usuário e para a dimensão semântica do produto, onde a forma segue o significado (KRIPPENDORFF, 1995).

De acordo com Bonsiepe (1999), o termo interface adquiriu seu significado atual a partir do surgimento dos computadores pessoais, na década de 1980 . Ainda segundo o autor, a importância do estudo de interfaces centradas no usuário cresceu na medida em que computadores deixaram de ser exclusividade de usuários especialistas, como matemáticos, físicos e engenheiros, e passaram a fazer parte da rotina diária de usuários que buscam, além de soluções para suas atividades profissionais, informação, comunicação e entretenimento. Como exemplo disso, o número de usuários de tecnologia móvel em 2012 era de 4,3 bilhões, com previsão de chegar a 5,2 bilhões até 2017 - ano em que smartphones, laptops e tablets serão responsáveis por 93\% do tráfego global de dados móveis (MURPHY, 2016; MADRUGA et al., 2015; BRADLEY et al., 2013) Moran (1981, p. 15) define interface como "a parte de um sistema computacional com a qual uma pessoa entra em contato - física, perceptiva ou conceitualmente". Para Levy (1999, p. 37), a interface permite a comunicação entre os sistemas de informação e comunicação, englobando "todos os aparatos materiais que permitem a interação entre o universo da informação digital e o mundo ordinário". Estudos recentes têm abordado a interface como a interpretação de códigos de máquina em um conjunto inteligível de estímulos multissensoriais, cuja efetividade leva em conta não só a funcionalidade, mas também os aspectos estéticos e emocionais (JOHNSON, 2001; BARDZELL, 2009; CHAPMAN, 2011; LOWDERMILK, 2013).

A facilidade com que o usuário identifica a funcionalidade de uma interface sem a necessidade de treinamento prévio, de forma a atingir metas específicas com eficácia, eficiência e satisfação é chamada de usabilidade (ISO, 1998). Jordan (1998) define esses três parâmetros da usabilidade da seguinte forma: eficácia refere-se à extensão na qual uma tarefa é realizada ou uma meta é alcançada; eficiência refere-se à quantidade de esforço empregado para se atingir a meta - quanto menor o esforço, maior será a eficiência; e satisfação refere-se ao nível de conforto que os usuários percebem quando utilizam o produto para atingir suas metas. Jordan (1998) ainda lista os princípios que devem nortear o designer que projeta interação: coerência, compatibilidade, capacidade, retroalimentação, prevenção, controle do usuário, clareza visual, priorização da funcionalidade, transferência adequada de tecnologia e evidências.

As tecnologias de informação e comunicação proporcionaram novas formas de colaboração em nível global, reinventando os tradicionais modelos de produção e distribuição de artefatos, informações e conteúdos. O design tem papel 
fundamental neste contexto, uma vez que a relação entre o homem e a tecnologia está cada vez mais presente no cotidiano dos mais variados perfis de usuários. O design de interação deve ser inclusivo, prevendo um público que vai de crianças a idosos novatos e avessos à tecnologia (ORPHANIDES; NAM, 2017). Diante disso, as próximas seções deste trabalho apresentam a análise bibliométrica do periódico Design Studies, com o objetivo de verificar se a produção científica em design nas últimas décadas tem refletido a emergência destas novas formas de interação entre usuário e tecnologia.

\section{PROCEDIMENTOS METODOLÓGICOS}

A técnica de pesquisa bibliométrica utiliza ferramentas estatísticas para quantificar um grande número de dados por meio do mapeamento e da geração de indicadores de tratamento e de gestão da informação (MENA-CHALCO; CESAR-JR, 2013; BUFREM; PRATES 2005). Por meio da análise dos dados é possível traçar um panorama da pesquisa sobre um tema específico, analisar o comportamento dos pesquisadores em suas decisões na construção do conhecimento, identificar os grupos mais representativos de pesquisadores em determinada área, bem como mapear a coautoria e as redes de cocitação.

O presente estudo teve o propósito de investigar a presença dos avanços tecnológicos nos artigos publicados no periódico Design Studies - Elsevier desde o seu surgimento. Para isso, foi realizada uma busca no banco de dados Scopus ${ }^{1}$ em outubro de 2015 por todos os artigos da revista, compreendendo o período de 1979 a 2015. No painel keywords do Scopus foram selecionadas apenas as palavras-chave relacionadas diretamente à tecnologia, possibilitando a exclusão de artigos que não atendiam a este critério de inclusão. A partir dos artigos selecionados, foram gerados gráficos por meio das ferramentas disponibilizadas pelo Scopus, como o número de artigos publicados por ano, por autor, por instituição de ensino e por país. Estes gráficos foram adaptados para uma melhor visualização dos dados. Gráficos adicionais foram desenvolvidos pelos autores deste estudo com base nos dados fornecidos pelo Scopus. Uma análise quantitativa foi realizada no gráfico de comparação entre o número de artigos sobre tecnologia em relação ao total de artigos publicados. Análises qualitativas foram realizadas por meio do ranqueamento dos artigos mais citados entre 1979 e 2015 e do levantamento das novas tecnologias que foram estudadas nesse período. Por fim, uma análise dos títulos e resumos dos artigos selecionados foi realizada por meio do software de análises bibliométricas VOSviewer (VAN ECK; WALTMAN, 2010),

1 Disponível em: <https://www.scopus.com>. 
com o objetivo de criar um mapa de visualização da rede de coocorrências dos termos mais relevantes dentro do domínio de interesse.

\section{RESULTADOS E DISCUSSÕES}

A Design Studies possui 962 artigos indexados na base de dados Scopus, que apresentam um total de 160 palavras-chave (acesso em 29 out. 2015). A partir da leitura das 160 palavras-chave, foi possível selecionar aquelas relacionadas à tecnologia, resultando em 41 palavras-chave (Figura 1). Uma busca utilizando as 41 palavras-chave selecionadas como filtro resultou em 526 artigos que abordam o tema tecnologia (Figura 2).

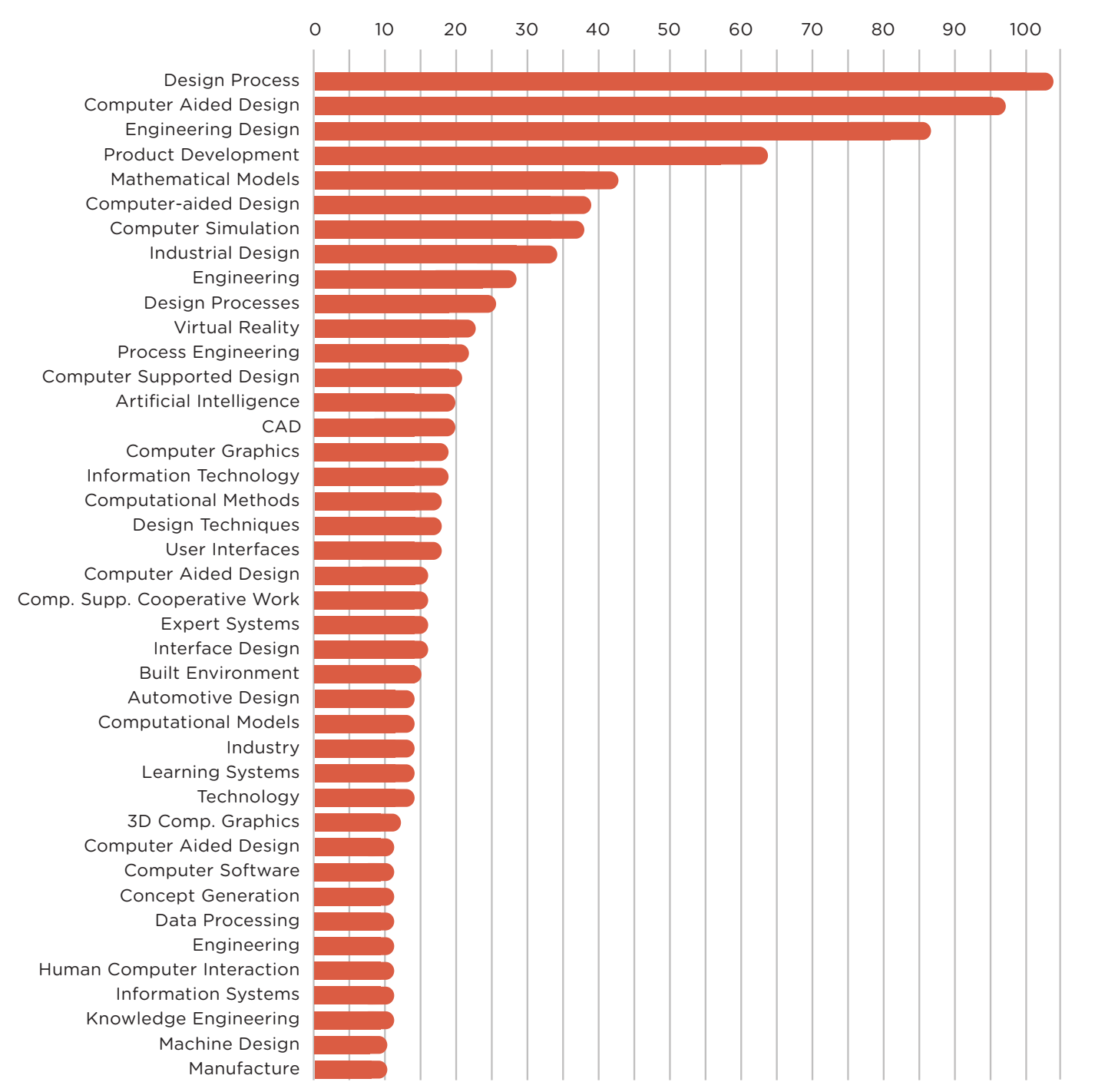

Figura 1: Número de ocorrências das 41 palavras-chave que mantém relação direta com o tema tecnologia. Fonte: elaborado pelos autores. 


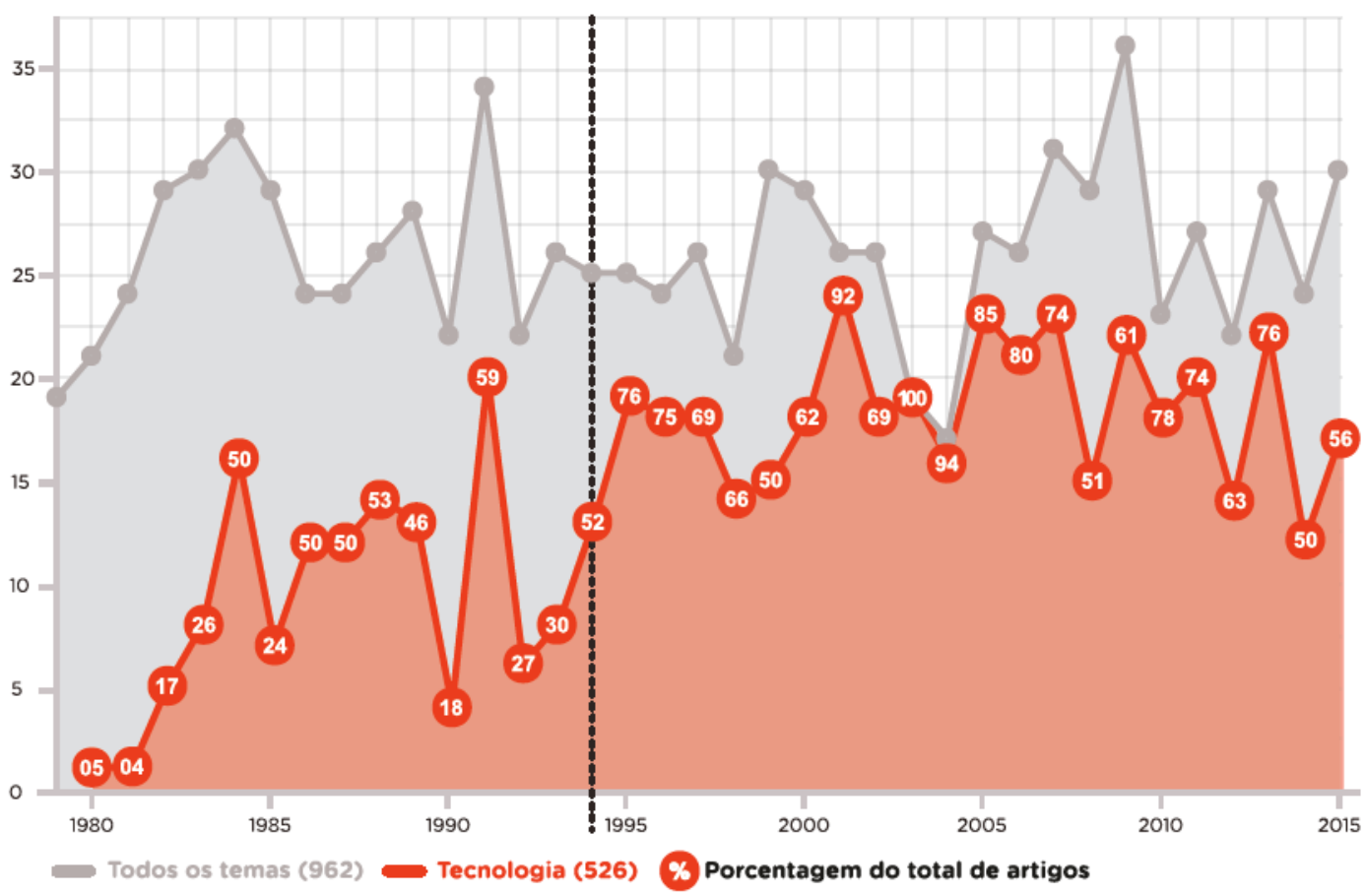

Figura 2: Proporçăo de artigos sobre tecnologia em relação ao total de artigos. Fonte: elaborado pelos autores.

\subsection{PUBLICAÇÕES POR ANO}

A Figura 2 revela que a proporção de artigos sobre tecnologia teve um aumento expressivo nos anos iniciais, entre 1980 e 1984, passando por breves momentos de queda em 1985, 1990 e 1992, retomando um crescimento regular nos anos subsequentes.

A partir de 1994 (linha tracejada na Figura 2) todas as edições da Design Studies passaram a contar com pelo menos $50 \%$ de seus artigos relacionados à tecnologia, o que evidencia uma consolidação do interesse dos pesquisadores pelo tema. A média nos 36 anos da revista é de $54,6 \%$.

\subsection{PUBLICAÇÕES POR PAÍSES, INSTITUIÇÕES E AUTORES}

Na lista dos dez países com mais artigos sobre o tema, o Reino Unido aparece em primeiro lugar, sendo responsável por $37,6 \%$ das publicações, seguido pelos Estados Unidos, com 21,4\%. Ambos concentram mais publicações que a soma de todos os outros países, com $59 \%$ de toda a produção mundial (Figura 3). No Reino Unido encontram-se quatro das 10 universidades que mais publicaram. Já os Estados Unidos concentram cinco dos 10 principais autores da lista. Com relação à representatividade das universidades, a Holanda destaca-se pela produção da Delft University of Technology que, apesar de não contar com os 
principais autores na área, é a universidade com maior número de publicações sobre o tema, com 29 artigos.

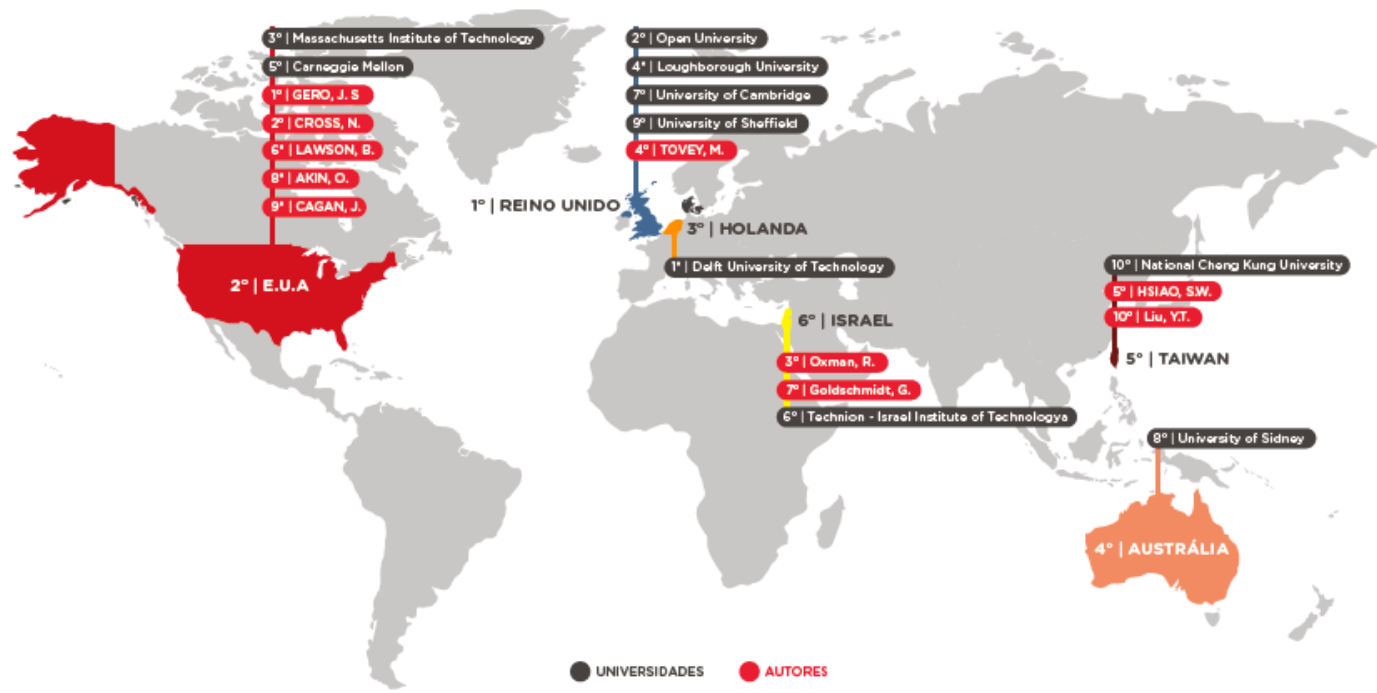

Figura 3: Ranking das principais instituiçóes e autores. Fonte: elaborado pelos autores.

As dez universidades e os dez autores que mais publicaram sobre o tema são originários dos seis países mais bem colocados na lista (Figura 4). Os outros quatro países juntos (Canadá, Dinamarca, Turquia e Suécia) representam apenas $8,3 \%$ do total de publicações.

Os autores americanos lideram a lista dos 20 artigos mais citados dentre os 526 retornados pela busca, com destaque para GERO, J. S., autor de 5 artigos da lista (Figura 5).

A leitura dos títulos e resumos dos artigos mais citados revela a predominância de temas relacionados a "design processes" e "design methods" (Figura 5). 19 artigos abordam a relação designer/tecnologia, enquanto apenas um artigo estuda a relação usuário/tecnologia. 

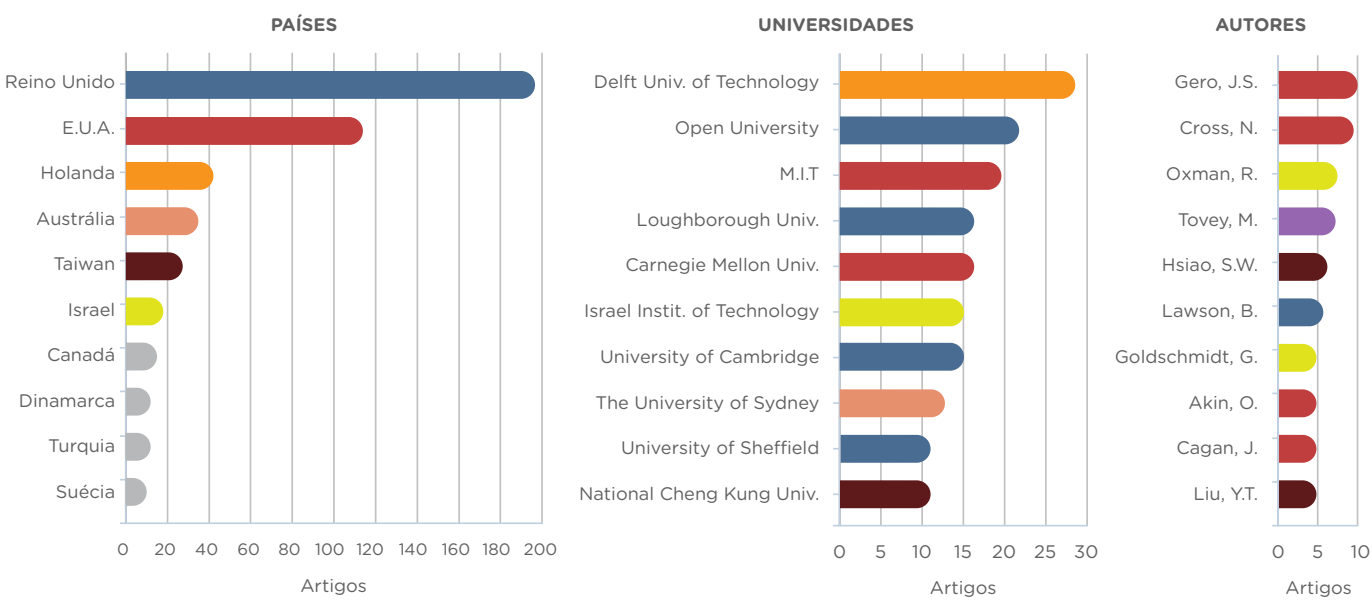

Figura 4: Número de publicações sobre tecnologia. Fonte: elaborado pelos autores.

Dorst, K., Cross, N. Creativity in the design process:
Co-evolution of problem-solution, 2001. Gero, J.S., Kannengiesser, U. The situated function-behaviour Jansson, D.G., Smith, S.M. Design fixation, 1991.

Stone, R.B., Wood, K.L., Crawford, R.H. A heuristic method for identifying modules for product architectures, 2000 Shah, J.J., Vargas-Hernandez, N., Smith, S.M. Metrics for measuring

Gero, J.S., Mc Neill, T. An approach to the analysis
of design protocols, 1998.

Purcell, A.T., Gero, J.S. Design and other types of fixation, 1996 Stempfle, J., Badke-Schaub, P. Thinking in design teams - An analysis of team communication, 2002. Dahmus, J.B., Gonzalez-Zugasti, J.P., Otto, K.N. Modular product architecture, 2001 . Suwa, M., Purcell, T., Gero, J. Macroscopic analysis of design processes Bucciarelli, L.L. An ethnographic perspective
on engineering design, 1998.

Howard, T. J. Culley, S. J., Dekoninck. E. Describing the creative design process by integration of engineering design and cognitive psychology, 2008 . Suwa, M., Gero, J., Purcell, T. Unexpected discoveries and S-invention of design McCormack, J.P., Cagan, J., Vogel, C.M. Speaking the Buick language: Capturing, .

Schön, D.A. Designing: Rules, types and words, 1988. Gross, M.D. The Electronic Cocktail Napkin - A computational environment for working with design diagrams, 1996 Chiu, M.-L. An organizational view of design communication Redström, J. Towards user design? on the shift from object to user as the subject of design, 2006 .
und Sonnenwald, D.H. Communication roles that support
collaboration during the design process, 2001 . Morelli, N. Product-service systems, a perspective shift for designers:
A case study, The design of a telecentre, 2003.

E.U.A. REINO UNIDO

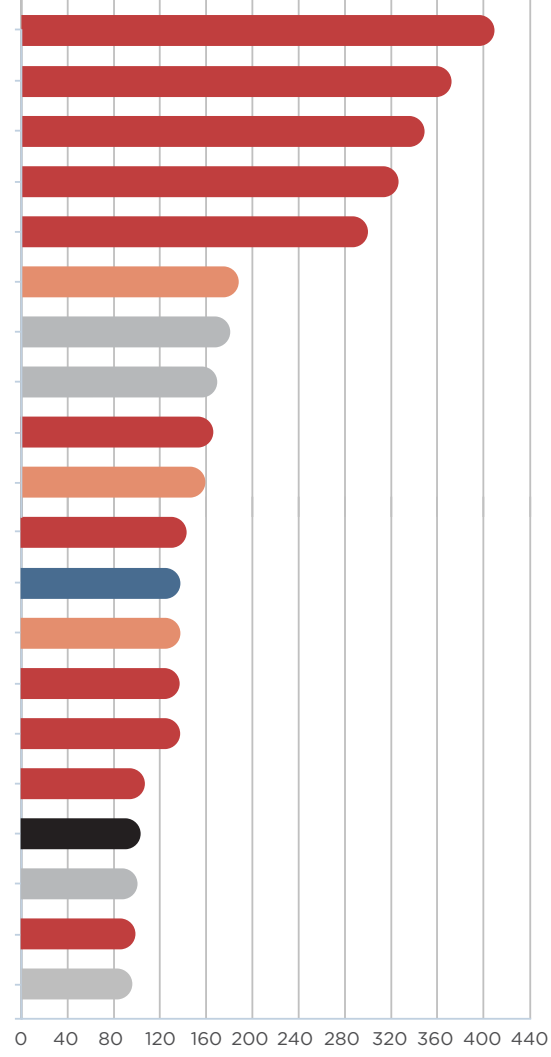

○ $40 \quad 80 \quad 120 \quad 160200240 \quad 280320360400440$

Figura 5: Artigos mais citados. Fonte: elaborado pelos autores.

5.3 NOVAS TECNOLOGIAS DE INFORMAÇÃO E DE COMUNICAÇÃO Tendo como base a revisão bibliográfica apresentada na seção 2 do presente estudo, uma nova busca foi realizada nos 526 artigos por termos relacionados às tecnologias que mais impactaram a sociedade informacional nas últimas 
décadas: novas tecnologias de informação e de comunicação. Os resultados possibilitaram uma análise mais detalhada da presença destas tecnologias nos artigos pesquisados.

O termo com mais ocorrências é "computer", que surge na Design Studies em 1980. No ano seguinte, a IBM lançou seu PC (personal computer), que se tornou sinônimo de computadores de uso pessoal. As ocorrências deste termo apresentaram um crescimento contínuo nos anos subsequentes, chegando a $61 \%$ de todos os artigos publicados em 2007, com uma média de 38,5\% do total de artigos publicados desde 1980 (Figura 6). O segundo termo com mais ocorrências é "interface", que aparece pela primeira vez em uma edição de 1983, apenas um ano após o lançamento do Macintosh da Apple, um personal computer com interface gráfica aprimorada (GUI - graphical user interface) que foi um sucesso de vendas por ter representado um grande avanço em termos de usabilidade (CURY; CAPOBIANCO, 2011). Em terceiro lugar está o termo "virtual", que surge em 1992 e atinge seu auge apenas em 2013, com 24\% do total de publicações. Artigos com os termos "internet", "web" e "online" vêm em quarto lugar, com um pico de $29 \%$ em 2005 e uma média 7,7\% de todos os artigos publicados desde sua primeira ocorrência, em 1987.

É possível afirmar, com base em uma contextualização histórica, que os termos "virtual", "internet", "web" e "online" tiveram um surgimento tardio na Design Studies. As primeiras experiências de conexão em rede entre computadores começaram na década de 1960 com objetivos militares e acadêmicos. A Internet continuou crescendo além de suas intenções iniciais graças ao surgimento de atividades comerciais online e à progressiva adoção por parte de usuários que não eram pesquisadores nem desenvolvedores. Em setembro de 1988 realizou-se o primeiro Interop Trade Show, oferecido por 50 empresas para mais de 5.000 engenheiros de corporações consideradas clientes potenciais (LEINER, 1997). Em 1989 foi criado o protocolo de comunicação especial chamado HTTP (HyperText Transfer Protocol), desenvolvido pelo pesquisador Timothy Berners-Lee, que culminou no projeto WWW (World Wide Web) (CURY; CAPOBIANCO, 2011). Nos artigos analisados, porém, referências ao uso de internet são ocasionais e começam a ganhar força apenas a partir de 1998, com uma defasagem de quase uma década. Em 2013, essas menções atingem seu auge, com $24 \%$ dos artigos publicados naquele ano (Figura 6). Os dispositivos móveis também representaram uma parcela muito pequena dos artigos analisados, com uma média de 1,7\% no período entre 1998 e 2015 . O resultado mais expressivo foi no ano de 2006 , quando $7,6 \%$ dos artigos publicados no periódico mencionaram os dispositivos móveis em sua temática (Figura 6). 


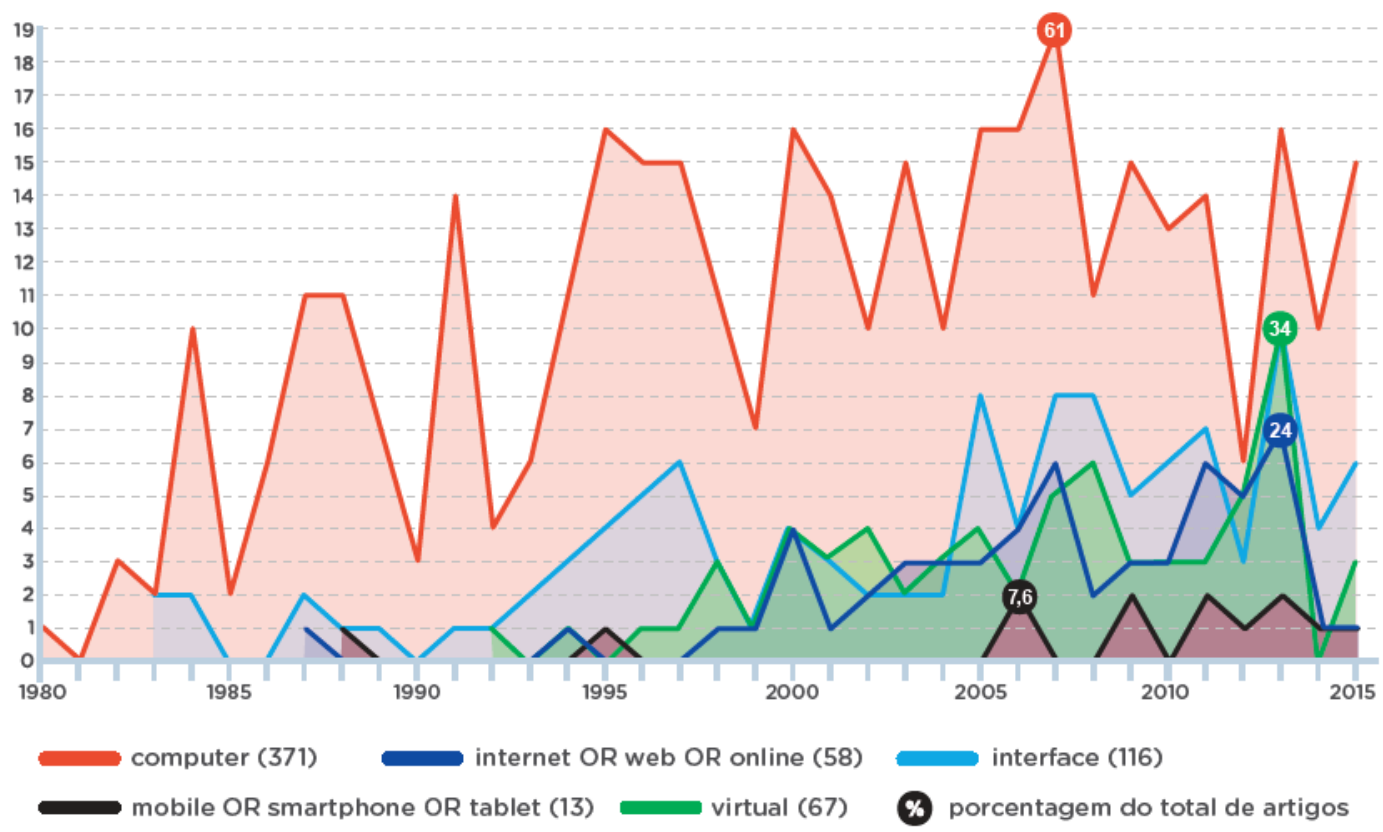

Figura 6: Novas tecnologias de informaçăo e comunicaçăo. Fonte: elaborado pelos autores.

\subsection{VISUALIZAÇÃO POR SIMILARIDADES}

Enquanto a contagem das palavras-chave oferece uma visualização quantitativa dos dados, visualizações do tipo vOS (visualization of similarities) possibilitam uma abordagem qualitativa, por identificarem a relevância dos termos com base em inter-relações. Por meio do software de análises bibliométricas VOSviewer (VAN ECK;WALTMAN, 2010), foram examinados os títulos e resumos dos 526 artigos selecionados. Para o VOSviewer, os termos (sintagmas nominais) que apresentam coocorrências com outros termos num padrão mais ou menos aleatório, são classificados como genéricos e recebem uma baixa pontuação de relevância. Por outro lado, se coocorrerem principalmente com um conjunto limitado de outros termos, são classificados como termos de significado mais específico e receberão uma alta pontuação de relevância. O VOSviewer permite deixar de fora os termos com baixa pontuação de relevância, eliminando, desta forma, termos genéricos.

A partir daí, é possível criar um mapa de visualização da rede de coocorrências dos termos de alta relevância dentro do domínio de interesse. Cada termo é representado por um círculo, cujo tamanho é definido pelo número de ocorrências enquanto as cores dos círculos indicam a proximidade em que os termos apareceram nos artigos. 


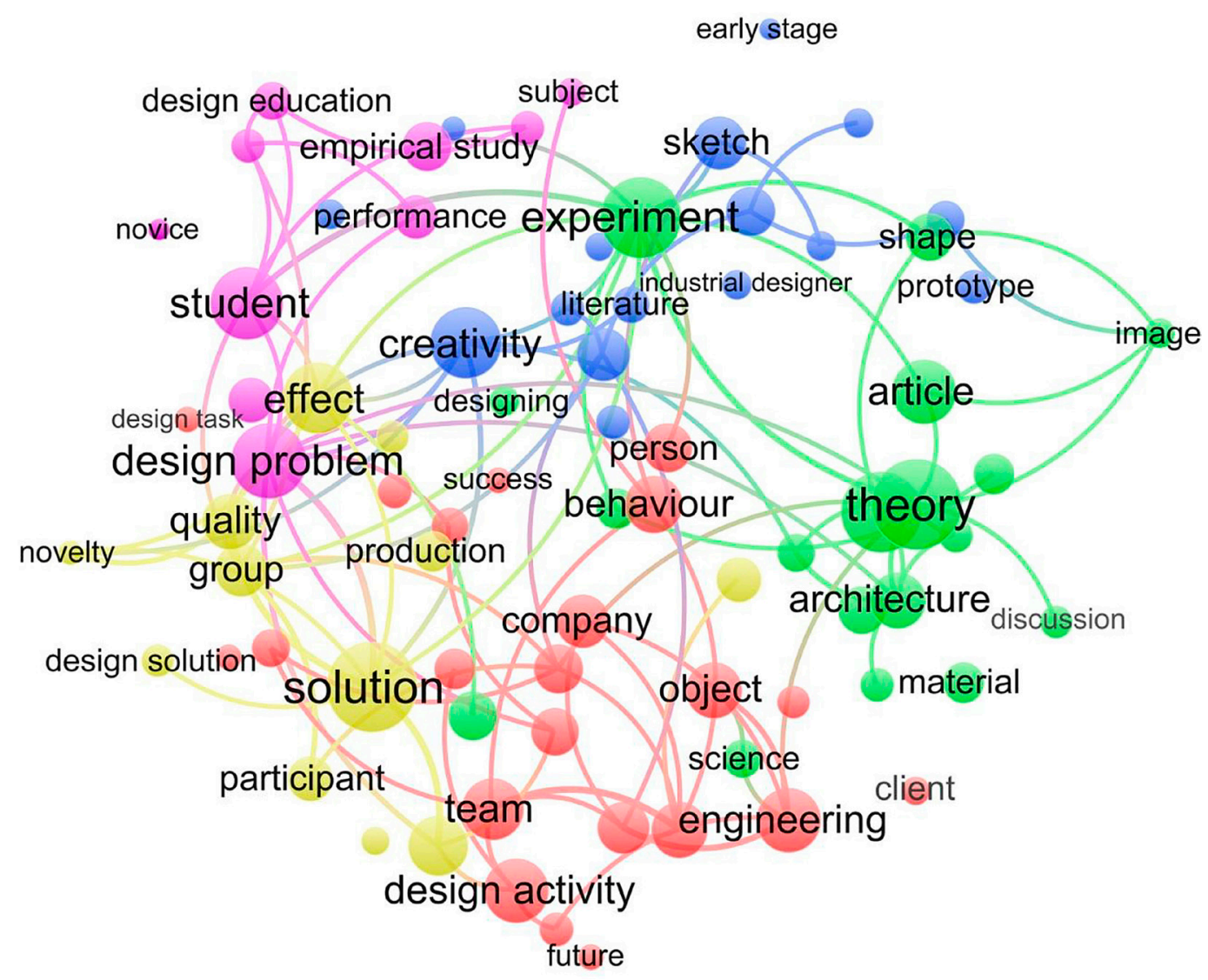

Figura 7: Visualização da rede de coocorrências dos termos de alta relevância. Fonte: elaborado pelos autores por meio do VOSviewer.

Utilizando o método de contagem binária (VAN ECK; WALTMAN, 2010), o software excluiu os termos com número de ocorrência menor que 10. Assim, dos 7.042 termos encontrados nos 526 artigos, apenas 193 tiveram ocorrência maior que 10. Para cada um destes 193 termos, uma pontuação de relevância foi calculada tendo como base os critérios de coocorrência e especificidade descritos anteriormente. Com o objetivo de favorecer a visualização dos termos, foram selecionados apenas os 60\% mais relevantes, resultando em uma rede de 116 termos (Figura 7). A Tabela 1 apresenta os termos com maior relevância resultantes da visualização por similaridades. 


\begin{tabular}{|l|l|l|}
\hline Termo & Ocorrências & Relevância \\
\hline early stage & 10 & 2.41 \\
\hline Novelty & 11 & 2.39 \\
\hline design student & 16 & 2.37 \\
\hline Image & 14 & 2.15 \\
\hline $\begin{array}{l}\text { CAD system } \\
\text { (computer aided design) }\end{array}$ & 14 & 1.97 \\
\hline
\end{tabular}

Tabela 1: Termos com maior relevância encontrados nos artigos sobre tecnologia.

Embora sejam os termos com o menor número de ocorrências da tabela, "early stage" e "novelty" apresentaram um grau de relevância maior por coocorrerem com um conjunto limitado de outros termos de significado mais específico. $\mathrm{O}$ único termo da lista que se refere à relação usuário/tecnologia é "client", e aparece em $15^{\circ}$ lugar, com relevância de 1.36 .

Estes resultados reafirmam os achados das Figuras 1 e 5, que indicam a predominância de estudos sobre a tecnologia como ferramenta do designer na fase projetual.

\section{CONSIDERAÇÕES FINAIS}

Na chamada sociedade informacional, as tecnologias de produção e informação estão cada vez mais presentes no cotidiano de bilhões de usuários, promovendo uma reorganização da produção e distribuição de artefatos, informações e conteúdos, impactando de forma radical as práticas profissionais, sociais e culturais.

Neste contexto, o design é o fator central da humanização destas tecnologias, se posicionando na intersecção entre a cultura da vida cotidiana, da tecnologia e da economia (BONSIEPE, 2012). Ao design cabe traduzir a tecnologia em uma linguagem que o usuário compreenda (JOHNSON, 2001), por meio de interfaces que permitam a interação com objetos, dados e processos.

A partir da análise bibliométrica realizada foi possível traçar um panorama da pesquisa sobre o tema, identificar os principais pesquisadores, seus respectivos campos de investigação e o papel destes estudos na construção do conhecimento na área do design. As universidades com maior pro- 
dução sobre o tema estão na Holanda e no Reino Unido, ao passo que os Estados Unidos concentram os autores com maior número de artigos e citações recebidas.

De modo geral, houve um constante crescimento do interesse dos pesquisadores por tecnologia, condizente com a importância progressiva que os avanços tecnológicos desempenharam na sociedade desde 1979 - ano em que o periódico teve início. Entretanto, a análise dos dados sugere que a Design Studies tem um perfil editorial voltado ao estudo da relação designer/tecnologia, em detrimento de estudos sobre a relação usuário/tecnologia. Houve uma prevalência de artigos com foco em processos e métodos, no uso da tecnologia pelo designer na fase projetual, na geração de ideias, na resolução de problemas e no trabalho em equipe.

As novas tecnologias de informação e de comunicação tiveram pouco destaque, se levarmos em conta a importância que desempenham nos dias atuais. Considerando que o número de usuários de smartphones, laptops e tablets em 2017 pode ultrapassar os 5 bilhões, o interesse dos pesquisadores por tais tecnologias parece não ter acompanhado este crescimento.

Além disso, artefatos e sistemas que antes não faziam uso de interfaces digitais hoje contam com estes recursos - como eletrodomésticos, eletroeletrônicos, sistemas de monitoramento, de segurança, de educação, de negócios - e estão cada vez mais presentes no cotidiano de bilhões de usuários no mundo, gerando uma demanda pela atuação de designers na relação do usuário com essas novas tecnologias. O design tem papel fundamental no suporte a esta interação, pois são necessárias interfaces adequadamente projetadas para públicos específicos, levando-se em conta, por exemplo, a inclusão digital, o crescimento da população idosa, a utilização de dispositivos por pessoas com deficiência, incapacidades ou mobilidade reduzida, entre outros fatores. Por isso, a pesquisa e a produção científica nesta área precisam ser ainda mais incentivadas para que seja possível atender a esta demanda.

\section{AGRADECIMENTOS}

Os autores agradecem à Coordenação de Aperfeiçoamento de Pessoal de Nível Superior - CAPES pelas bolsas de mestrado demanda social.

\section{REFERÊNCIAS BIBIOGRÁFICAS}

ASHTON, Kevin. That 'Internet of Things' thing. 2009. Disponível em: <http:// www.rfidjournal.com/article/view/4986>. Acesso em: 8 out. 2015. 
BARDZELL, Jeffrey. Interaction criticism and aesthetics. In: Proceedings of the SIGCHI Conference on Human Factors in Computing Systems. ACM, 2009. p. 2357-2366.

BONSIEPE, Gui. Design: como prática de projeto. São Paulo: Blucher, 2012.

BONSIEPE, Gui. Design: from material to digital and back. In: Interface, An Approach to Design, Maastricht: Jan van Eyck, 1999.

BRADLEY, Joseph et al. Winning the New Digital Consumer with Hyper-Relevance: In Retail, Insight Is Currency and Context Is King. 2015. Disponível em: <http://www.cisco.com/c/dam/en/us/solutions/collateral/executive-perspectives/ioe-retail-whitepaper.pd> Acesso em: 8 out. 2015.

BROCK, David L. The Electronic Product Code (EPC): A naming Scheme for Physical Objects. 2001. Disponível em: <http://cocoa.ethz.ch/downloads/2014/06/None_MIT-AUTOID-WH-002.pdf >. Acesso em: 8 out. 2015.

BUFREM, Leilah; PRATES, Yara. $\mathbf{O}$ saber científico registrado e as práticas de mensuração da informação. Ciência da Informação, Brasília, v. 34, n. 2, p. 9-25, 2005. Disponível em: <http://www.scielo.br/pdf/ci/v34n2/28551>. Acesso em: 12 ago. 2010.

BURDEA, Grigore C.; COIFFET, Philippe. Virtual RealityTechnology. London: Wiley-Interscience, 1994.

CHAMBERS, John. Internet of Everything: Fueling an Amazing Future \#TomorrowStartsHere. 2012. Disponível em: <http://blogs.cisco.com/news/internet-of-everything-2>. Acesso em: 8 out. 2015.

CHAPMAN, Stephen J. Design de Interação: além da Interação Humano-Computador. Porto Alegre, Bookman Editora, 2011.

CURY, Lucilene; CAPOBIANCO, Ligia. Princípios da História das Tecnologias da Informação e Comunicação Grandes Invenções. In: VIII Encontro Nacional de História da Mídia. Anais... Guarapuava: Unicentro, 2011. p. 1-13.

DE MASI, Domenico. O Ócio Criativo. Tradução de Léa Manzi. Rio de Janeiro: Sextante, 2000.

EVANS, Dave. A Internet das Coisas: como a próxima evolução da Internet está mudando tudo. 2011. Disponível em: <http://www.cisco.com/web/BR/ assets/executives/pdf/internet_of_things_iot_ibsg_0411final.pdf $>$. Acesso em: 8 out. 2015.

EVANS, Dave. Answering the Two Most-Asked Questions About the Internet of Everything \#loE. 2013. Disponível em: <http://blogs.cisco.com/ioe/ answering-the-two-most-asked-questions-about-the-internet-of-everything $>$. Acesso em: 8 out. 2015. 
MENA-CHALCO, Jesús P; CESAR-JR, Roberto M. Bibliometria e cientometria: reflexões teóricas e interfaces. São Carlos: Pedro \& João Editores, 2013.

IERC (EUROPEAN RESEARCH CLUSTER ON THE INTERNET OF THINGS). IoT Semantic Interoperability: Research Challenges, Best Practices, Recommendations and Next Steps. 2015. Disponível em: <http://www.internet-of-things-research.eu/pdf/IERC_Position_Paper_loT_Semantic_Interoperability_Final. pdf > . Acesso em: 8 out. 2015.

ICSID (INTERNATIONAL COUNCIL OF SOCIETIES OF INDUSTRIAL DESIGN). Definition of Design. Disponível em: <http://wdo.org/about/definition/>. Acesso em: 10 set. 2015.

ISO. ISO 9241-11: Ergonomic requirements for office work with visual display terminals (VDTs): Guidelines for specifying and measuring usability. Genebra: International Organization for Standardization, 1998.

ITU (INTERNATIONAL TELECOMMUNICATION UNION). ITU Internet Reports 2005: The Internet of Things. 2005. Disponível em: <http://www.itu.int/osg/ spu/publications/internetofthings $>$. Acesso em: 8 out. 2015.

JOHNSON, Steven. Cultura da interface: como o computador transforma nossa maneira de criar e comunicar. Rio de Janeiro: Jorge Zahar Editora, 2001.

JORDAN, PatrickW. An Introduction to Usability. Londres: Taylor \& Francis, 1998. KRIPPENDORFF, Klaus. On the essential contexts of artifacts or on the proposition that "design is making sense (of things)". Design Issues, v. 5, n. 2, p. 9-39, 1989.

LEINER, Barry M. et al. The past and future history of the Internet. Communications of the ACM, v. 40, n. 2, p. 102-108, 1997.

LÉVY, Pierre. Cibercultura. Tradução de Carlos Irineu Costa. São Paulo: Editora 34, 1999.

LIPOVETSKY, Gilles; SERROY, Jean. A cultura-mundo: resposta a uma sociedade desorientada. Tradução de Maria Lúcia Machado. São Paulo: Companhia das Letras, 2011.

LOWDERMILK, Travis. Design centrado no usuário: um guia para o desenvolvimento de aplicativos amigáveis. São Paulo: Novatec Editora, 2013.

MADRUGA, Ewerton L. et al. Assessing Quality of Experience while comparing competing mobile broadband services from the user perspective. Journal of Physics: Conference Series. IOP Publishing, 2015. p. 01, 2012.

McGARRY, Kevin. MCGARRY, Kevin. $\mathbf{O}$ contexto dinâmico da Informação: uma análise introdutória. 2. ed. Brasília: Briquet de Lemos, 1999. 
MORAN, Thomas P. The Command Language Grammars: a representation for the user interface of interactive computer systems. International Journal of Man-Machine Studies, n. 15, p. 3-50, 1981.

MURPHY, Matt; MEEKER, Mary. Top mobile internet trends. KPCB Relationship Capital, 2011. Disponível em: <http://www.kpcb.com/blog/top-10-mobile-internet-trends $>$. Acesso em: 4 mar. 2016.

NORMAN, Donald A. 0 design do dia-a-dia. Tradução de Ana Deiró. Rio de Janeiro: Rocco, 2006.

ORPHANIDES, Andreas K.; NAM, Chang S. Touchscreen interfaces in context: A systematic review of research into touchscreens across settings, populations, and implementations. Applied Ergonomics, v. 61, p. 116-143, 2017. RIBEIRO, Vinicius G.; SILVEIRA, André da. Sociedade e tecnologia: uma relação complexa. In: MENEZES, M. S., MOURA, M. (Org). Rumos da Pesquisa no Design Contemporâneo: relação tecnologia $x$ humanidade. 1. ed. São Paulo: Estação das Letras e Cores, 2013, p. 358-371.

SINGER, Talita. Tudo conectado: conceitos e representações da internet das coisas. In: Simpósio em tecnologias digitais e sociabilidade, 2., 2012, Salvador. Anais... Salvador: UFBA, 2012. p. 1-15.

VAN ECK, Nees J.; WALTMAN, Ludo. Software survey: VOSviewer, a computer program for bibliometric mapping. Scientometrics, v. 84, n. 2, 2010. 
Luis Carlos Paschoarelli é Professor Titular no Departamento de Design da UNESP (2017), Bolsista de Produtividade em Pesquisa do CNPq - Nível 1D - CA DI - Desenho Industrial e Livre-Docente em Design Ergonômico pela Universidade Estadual Paulista (2009). Possui pós-doutorado em Ergonomia (2008) pela Universidade Técnica de Lisboa, doutorado em Engenharia de Produção (2003) pela UFSCar, mestrado em Projeto, Arte e Sociedade - Desenho Industrial (1997) e graduação em Desenho Industrial (1994) pela UNESP. É co-líder no Grupo de Pesquisa Design Ergonômico: Projeto e Interfaces onde coordena os projetos de pesquisa: Design Ergonômico: avaliação e intervenção ergonômica no projeto; Design Ergonômico: metodologias para a avaliação de instrumentos manuais na interface homem x tecnologia; e Contribuições do Design Ergonômico na pesquisa e projeto de equipamentos para a reabilitação de pessoas com capacidades específicas. Está lotado no Laboratório de Ergonomia e Interfaces Departamento de Design, onde atua como docente no curso de graduação em Design e no Programa de Pós-graduação em Design da UNESP. Tem experiência na área do design, ergonomia, design ergonômico, design de produto e design gráfico. Atualmente é coordenador do Programa de Pós-Graduação em Design - Mestrado e Doutorado - da UNESP e é "Ergonomista Sênior" da ABERGO - Associação Brasileira de Ergonomia.

Fabrício Mira é Bacharel em Desenho Industrial com Habilitação em Programação Visual pela UNESP (2006) e mestre pelo Programa de Pós-Graduação em Design - Linha de Pesquisa Planejamento de Produto pela mesma universidade (2017). Membro do grupo de Pesquisa em Design Contemporâneo: sistemas, objetos e cultura. Tem experiência nas áreas de Design e Artes, com ênfase nos seguintes temas: Design Gráfico, Identidade Visual, Ergonomia, Artes Plásticas, Ilustração Digital 2D/3D, Ilustração Não-Digital, Realidade Virtual e Histórias em Quadrinhos. Atuou como professor de ensino superior na Graduação do curso de Artes e na Pós-Graduação do curso de Letras e Artes - Interlinguagens do Texto e da Imagem pelas Faculdades de Dracena/SP, mantidas pela Fundação Dracenense de Educação e Cultura - FUNDEC. Possui publicações em capítulo de livro e anais de congresso como "A Ergonomia aplicada ao design de jornais - um estudo de caso dos jornais de Bauru- SP" (In: Estudos em Design nas Universidades Estaduais Unesp e Usp. 1 ed. São Paulo: Editora UNESP, 2006. v. 1. 308p.) e "Contribuições do Design para o uso de realidade virtual no tratamento da síndrome do pânico" (In: IDEMI 2015 - IV Internacional Conference on Integration of Design, Engeneering and Management for Innovation, 2015, Florianópolis/SC). 
Lílian Lago é Bacharel em Design Gráfico (2010) e especialista em Gestão Estratégica de Design (2012) pela Universidade Estadual de Londrina e mestre pelo Programa de Pós-Graduação em Design - Linha de Pesquisa Planejamento de Produto da UNESP (2017). Membro do Grupo de Pesquisa Design Contemporâneo: sistemas, objetos e cultura. Tem experiência em design, com ênfase em desenvolvimento de produtos, estratégia e design editorial. Foi designer da Editora da Universidade Estadual de Londrina e analista de negócios em design, tecnologia e inovação no SENAI Paraná. Possui publicações como os capítulos de livro "Ensino e Pesquisa Científica no Design e na Moda no Brasil: caminhos que se cruzam e se realimentam" (In: Maria de Fátima da Silva da Costa Mattos. (Org.). Pesquisa e formação em Moda. 1ed. São Paulo: Estação das Letras e Cores, 2015) e "Design Contemporâneo: pesquisas interdisciplinares" (In: Luis Carlos Paschoarelli e Rosio Fernández Baca Salcedo. (Org.). Interação: panorama das pesquisas em Design, Arquitetura e Urbanismo. 1ed. Bauru: Canal 6, 2015), artigos publicados em periódicos como "A Pesquisa em Design e a Interdisciplinaridade" (In: Revista Educação Gráfica, v. 19, p. 106-123, 2015) e artigos publicados em anais de eventos como "Design and fashion processes and methods in the contemporary scene" (In: $3^{\circ}$ CIMODE - Congresso Internacional de Moda e Design, 2016, Buenos Aires, Argentina).

Juliana Cristina de Angelo é Graduada em Educação Física pela Faculdade de Ciências da UNESP/ Bauru (2006), e mestre pelo Programa de Pós-Graduação em Design - Linha de Pesquisa Ergonomia pela mesma universidade (2017). Membro do grupo de pesquisa do Laboratório de Informação, Visão e Ação (LIVIA), liderado pelo Prof. Dr. Sérgio Tosi Rodrigues. Tem experiência em Educação Física. Como educador físico trabalha com reabilitação física e condicionamento e nos seguintes temas: movimento dos olhos, controle postural e condução simulada de veículos. Possui publicações em congresso e apresentações como "Influences of open and closed on posture balance body during the range of an object"' e "A percepção do usuário no âmbito da Ergonomia: um estudo bibliométrico" (In: IDEMI 2015 - IV Internacional Conference on Integration of Design, Engeneering and Management for Innovation, 2015, Florianópolis/SC). Apresentações em congresso como: "Comportamento dos olhos e da postura em adultos jovens" (In: VII Congresso Brasileiro de Comportamento Motor, Belo Horizonte/MG, 2014), "Movimento dos olhos em diferentes bases de apoio em adultos jovens e crianças" (In: 40 Encontro Paulista de Comportamento Motor, Ribeirão Preto/SP, 2015), "Influências das posturas abertas e fechadas sobre o 
controle postural" na modalidade de painel científico (In: V Simpósio do Programa de Pós-Graduação em Psicologia do Desenvolvimento e Aprendizagem, Faculdade de Ciências da UNESP/Bauru, 2015).

Recebido em: 29/01/2017

Aceito em: 29/03/2017 